\title{
Geometry optimization in basic CMOS cells for improved power, leakage, and noise performances
}

\author{
Javier Castro, Antonio J. Acosta
Instituto de Microelectrónica de Sevilla/
University of Seville, Seville, Spain \\ e-mail: \{casram,acojim\}@imse.cnm.es
}

\begin{abstract}
The rising demand for portable system is increasing the importance of low power as a design consideration. In this sense, leakage power is increasing much faster that dynamic power at smaller dimensions. Peak values of supply current are related to noise injected into the substrate and/or propagated through supply network, limiting the performances of the sensitive analog and RF portions of mixed-signal circuits. This paper analyses how these three aspects, dynamic power, leakage power and peak power, can be considered together, optimizing the sizing and design of basic cells, with a reduced degradation in performances. The suited sizing of basic cells, show the benefits of the proposed technique, validated through simulation results on $130 \mathrm{~nm}$ nand, nor and inverter cells.
\end{abstract}

\section{Introduction}

Power consumption and power-related issues have become a first-order concern for most designs, because of the rising demand for portable system [1-3]. A lot of techniques for low power design of VLSI circuits targeting both dynamic and leakage components of power dissipation in CMOS VLSI circuits have been recently presented [1-3]. The primary method used to date for reducing power has been supply voltage $\left(\mathrm{V}_{\mathrm{DD}}\right)$ reduction, although this technique begins to lose its effectiveness as voltages drop to sub-one volt range and further reductions in the supply voltage begin to create more problems than are solved [1-3]. For this reason, it is important the optimization of geometries for cells at an specific $\mathrm{V}_{\mathrm{DD}}$.

A well known expression [1] for the power consumed in a CMOS circuit is shown in eq. (1).

This work has been sponsored by the Spanish MEC TEC2007-65105 TICOCO and the Junta de Andalucía TIC2006-635 Projects

\author{
Mark Vesterbacka
Dept. of Electrical Enginnering, Linköping University, Linkoping, Sweden \\ e-mail: markv@isy.liu.se
}

$P=P_{D y n}+P_{S C}+P_{L k}=\frac{1}{2} a C_{L} f V_{D D}^{2}+V_{D D} I_{S C}+V_{D D} I_{L k}^{(1)}$

Eq. (1) includes dynamic, short circuit and leakage power. In the same way, power is related to the supply current consumption curve: the average power, defined as the (dis)charge (dynamic) current plus the short circuit current, the leakage current. Besides this, the maximum value of supply current for a given pattern, known as peak current, is traditionally indirectly related to switching or didt noise [4,5]. being the cause of the limitation of the performances of the sensitive analog and RF portions of mixed-signal circuits.

This paper proposes a simulation-based optimization procedure, allowing the achievement of geometries for basic cells, providing reduced values of peak current and both dynamic and leakage power consumption.

The organization of the paper is as follows: Section 2 analyzes the supply current related issues; Section 3 presents the optimization methodology; Section 4 includes demonstrative simulation results. Finally, the main conclusions are presented.

\section{Supply current related issues}

Lot of work has been done to establish precise models for the power consumption in their different components. In most cases, not analytical but empirical model are achieved because of the multifactorial and technological dependencies. We are interested in analysing and exploiting their dependencies on $\mathrm{V}_{\mathrm{DD}}$ and transistor sizes. A brief discussion on this is shown below.

\subsection{Peak of supply current}

The maximum value of supply current $\left(\mathrm{I}_{\text {peak }}\right)$ shows multiple dependencies on output load, input-output coupling capacitance and input slope, bringing as a consequence several empiric models for $\mathrm{I}_{\text {peak }}[6,7]$. 
Considering a CMOS inverter under the assumption that the gate has been sized such that the pull-up and pull-down network transconductances are roughly equal, the peak current in the load device will occur at a gate voltage of approximately $\mathrm{V}_{\mathrm{DD}} / 2$. At this time, the load transistor will experience a small drain-to-source voltage which has been caused by the active transistor which begins switching the capacitive load once the input voltage exceeds its threshold voltage. In nanometric devices, the saturation voltage is typically much smaller than the expected long-channel value of $\mathrm{V}_{\mathrm{GS}^{-}}$ $\mathrm{V}_{\mathrm{TH}}$. When $\mathrm{V}_{\mathrm{GS}}=\mathrm{V}_{\mathrm{DD}} / 2$, we expect that a drain-tosource voltage in the range of $\left(\mathrm{V}_{\mathrm{GS}}-\mathrm{V}_{\mathrm{TH}}\right) / 2 \sim\left(\mathrm{V}_{\mathrm{DD}} / 2-\right.$ $\left.\mathrm{V}_{\mathrm{DD}} / 4\right) / 2=\mathrm{V}_{\mathrm{DD}} / 8$ will be sufficient to saturate the load device. This approximation allows to estimate the peak current at $\mathrm{V}_{\mathrm{GS}}=\mathrm{V}_{\mathrm{DD}} / 2$. Based on the alpha-power law model [8] for $\mathrm{I}_{\mathrm{DS}}$, it can be determined (2):

$I_{\text {peak }}=\left(\frac{V_{G S}-V_{T H}}{V_{D D}-V_{T H}}\right)^{\alpha} I_{D S, \text { Sat }}$

being $\mathrm{I}_{\mathrm{DS} \text {,Sat }}$ calculated as the following empirical formulation for deep submicron devices [9], as (3):

$I_{D S, S a t}=\frac{1}{2} \frac{W}{L} \mu_{0} C_{o X}\left(V_{G S}-V_{T H}\right)^{\alpha}$

When substituting nominal approximated value for $\mathrm{V}_{\mathrm{GS}}\left(\mathrm{V}_{\mathrm{DD}} / 2\right), \alpha(1.3), \mathrm{V}_{\mathrm{DD}}(1.2 \mathrm{~V})$ and $\mathrm{V}_{\mathrm{TH}}(=0.265 \mathrm{~V})$, yields the expression in (4) for the peak in supply current, roughly $24 \%$ of the device's saturation current, a linear dependence with W/L and a non-linear dependence with supply voltage $\mathrm{V}_{\mathrm{DD}}$.

$I_{\text {peak }} \propto \frac{\left(V_{D D} / 2-0.265\right)^{2.6}}{\left(V_{D D}-0.265\right)^{1.3}} \frac{W}{L}$

It should be noticed that this value could fluctuate greatly depending on the output load of the gate and the input slope. For instance, a very small load at the output of the gate would mean that the output voltage can swing more quickly, such that the load transistor will be even deeper in the saturation regime; leading to a larger current. On the other hand, with a large load and a quick input transition, the output will not have had time to switch very significantly keeping in the linear region of operation. However, when the load is moderate, equations (2) and (3) yield adequate representations of the load device current. It was noted in [8] that shortchannel devices exhibit more short-circuit current than long-channel MOSFET's. This is due to the fact that the device saturates at a smaller drain-to-source voltage, allowing for a larger current to flow in the time interval corresponding to the transition. In this analysis, the effects of resistive-capacitive-inductive supply distribution network have been neglected for simplicity.

\subsection{Leakage Power}

In many new high performance designs, the leakage component of power consumption is higher than the switching component (up to $70 \%$ or even higher percentage) [1-3]. This percentage will increase with technology scaling unless effective techniques are introduced to bring leakage under control.

There are four main sources of leakage current in a MOS transistor:

1. Reverse-biased junction leakage current (IREV)

2. Gate induced drain leakage (IGIDL)

3. Gate direct-tunnelling leakage $(I G)$

4. Subthreshold leakage (ISUB)

For current technologies, the ISUB is the dominant component among the four components of $\mathrm{I}_{\text {leakage }}[1]$. A usual expression for ISUB is presented in eq. (5), where $V t$ is the thermal voltage, $n$ is an experimental value, and $\eta$ accounts for the DIBL effect, that will be neglected for simplicity $(\eta=0)[1]$.

$$
I_{S U B}=\mu_{0} C_{O X} V_{t}^{2} \frac{W}{L} \exp \left(\frac{V_{G S}-V_{T H}+\eta V_{D S}}{n V_{t}}\right)\left(1-\exp \left(-\frac{V_{D S}}{V t}\right)\right)
$$

As clearly stated from (5), leakage linearly depends on $\mathrm{W} / \mathrm{L}$, and exponentially on $\left(\mathrm{V}_{\mathrm{GS}}-\mathrm{V}_{\mathrm{TH}}\right)$ and $-\mathrm{V}_{\mathrm{DS}}$.

\subsection{Dynamic Power}

Dynamic (average) power consumption in CMOS circuits consists primarily of short circuit dissipation and the switching power consumed while charging and discharging load capacitances, according to eq. (1). The dynamic power is usefully expended, since it is associated with the switching of logic states that is central to performing logic operations. (Dis)charge power is proportional to $C V_{\mathrm{DD}}^{2} f$, where $C$ is the capacitance, $V_{\mathrm{DD}}$ is the supply voltage, and $f$ is the clock frequency. This power dissipation is in direct proportion to the rate of computation, and so can be adjusted to meet application power requirements by adjusting the computation rate. It can also be adjusted, to a more limited extent, by adjusting the supply voltage. The dependence of (dis)charge power on $\mathrm{W} / \mathrm{L}$ is related to the value of parasitic capacitance $\mathrm{C}$ associated to the gate, being proportional to $\mathrm{W} / \mathrm{L}$. The short circuit power strongly depends on the time that both pull-up and pull-down are in simultaneous saturation state (input slope), being proportional to $\mathrm{W} / \mathrm{L}$ ratio and $\left(\mathrm{V}_{\mathrm{GS}}-\mathrm{V}_{\mathrm{TH}}\right)^{\alpha}$, as it is stated in eq. (3). 


\section{Optimization technique}

It is clear from the previous section, that a linear dependence of $I_{\text {peak }}$, $I_{\text {avg, and }} \mathrm{I}_{\text {leakage }}$ on W/L can be considered in a first approach. In the same way, a non linear dependence with $\mathrm{V}_{\mathrm{DD}}$ is also clearly stated: quadratic for dynamic power considering (dis)charge current, but polynomial for short circuit, peak power and leakage.

Additionally, the propagation delay associated to a simple CMOS cell can be evaluated [8] as eq. (6), where the linear (inverse) dependence on $\mathrm{W} / \mathrm{L}$, the linear dependence on load capacitance $\mathrm{C}_{\mathrm{L}}$ and polynomial dependence on $\mathrm{V}_{\mathrm{DD}}$ are clearly stated. Parameter $\alpha$ is 1.3 for the selected technology.

$$
\text { Delay }=\frac{C_{L} V_{D D}}{\mu_{0} C_{o x} \frac{W}{L}\left(V_{D D}-V_{T H}\right)^{\alpha}} \text { with } \alpha \in(1,2)
$$

The main idea is to get benefits from these dependencies in order to optimize the suited selection of geometries and supply voltage for basic cells. A reduction of $\mathrm{V}_{\mathrm{DD}}$ produces, as immediate consequence, the reduction of the different components of power, but increasing the delay. However the increment in delay can be compensated if the width of the transistors is increased in the same proportion. This increment in the width also produces a quasilinear increment in components of power, so a trade-off between area, delay and power components (leakage, peak and average) can be found.

The proposal is to increment the width of the transistor applying a correction factor fw, obtained after a parametric analysis, as the supply voltage is reduced by a factor $f v d d$, maintaining the propagation delay almost constant, keeping reduced the overhead in power parameters.

To do this, the iterative scheme in Fig. 1 is considered. The start point of the procedure is the selection of the cells and transistor's dimensions, nominally minimum width and length. The gate is simulated and characterized for the selected technology, under the nominal supply voltage $(f v d d=1)$. The propagation delay, measured as the average of high to low and low to high delays $(\mathrm{tp}=(\mathrm{tphl}+\mathrm{tplh}) / 2)$, the leakage current $\left(\mathrm{I}_{\text {leakage }}\right)$, the peak $\left(\mathrm{I}_{\text {peak }}\right)$ and the average $\left(\mathrm{I}_{\mathrm{avg}}\right)$ of supply current are measured by SPECTRE simulation. Once characterized, the fvdd parameter is reduced, and a parametric analysis is performed to obtain a value of $f w$, modifying the width of the transistors of the gates in a factor Wfw, in such a way that the propagation delay for this choice of $f w$ is approximately the same (within $0.1 \%$ of tolerance) than that obtained for $f v d d=1$. The length of the

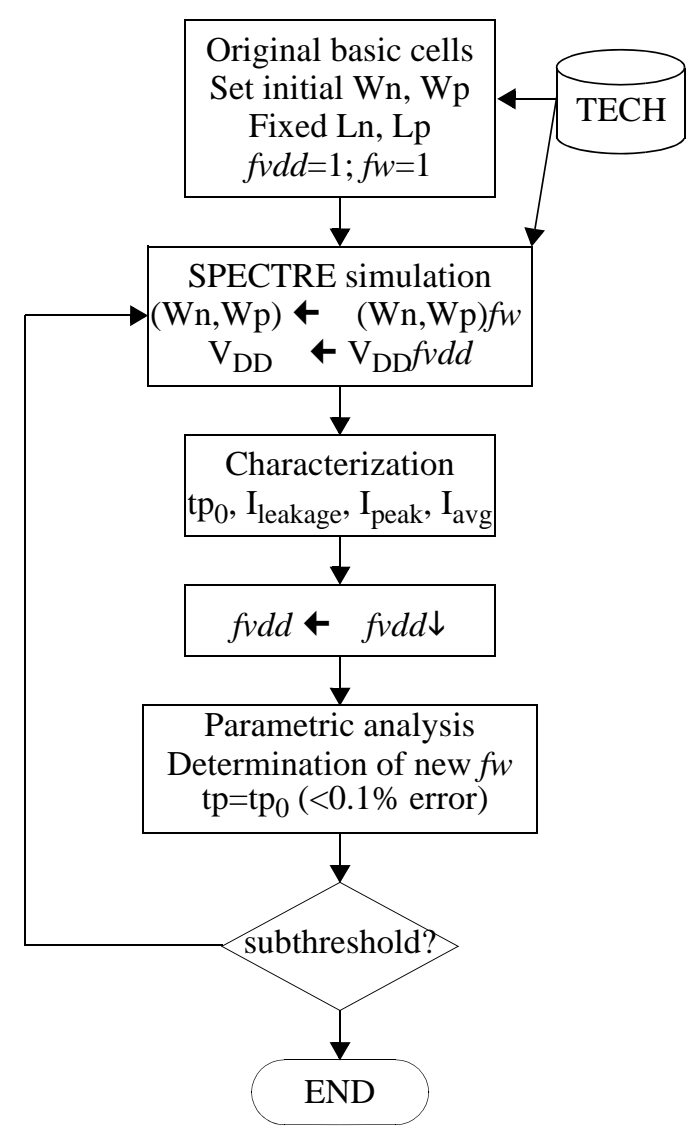

Figure 1. Flow diagram of the optimization procedure.

transistors are kept constant. The process is repeated with different values of $f v d d$, until the supply voltage is reduced down to a half of its initial value, in order to the transistors operate out of the subthreshold region. In our case we have considered four different values for fvdd and obtained the corresponding $f w$ values, saving power and reducing peaks in supply current, as it will be shown in next section.

\section{Simulation results}

To perform the SPECTRE simulations needed to run the procedure explained in the previous section, the simulation set-up of Fig. 2 has been considered. The basic cells selected for evaluating the procedure are the basic CMOS inverter, the 2-input Nand and the 2-input Nor gates, as shown in Fig. 2. The output inverters are used to simulate realistic load conditions for the cell under study. The input patterns are square $100 \mathrm{MHz}$ waveforms, with 25ps of transition times. The selected technology was UMC $130 \mathrm{~nm}$, with a nominal supply voltage of $1.2 \mathrm{~V}$. Dimensions are the minimum for the technology: $\mathrm{Wn} / \mathrm{Ln}=0.15 / 0.12$ um (N1 and N3 in Fig. 2) and $\mathrm{Wp} / \mathrm{Lp}=0.3 / 0.12$ um (P1 and P2 in Fig. 2), being 


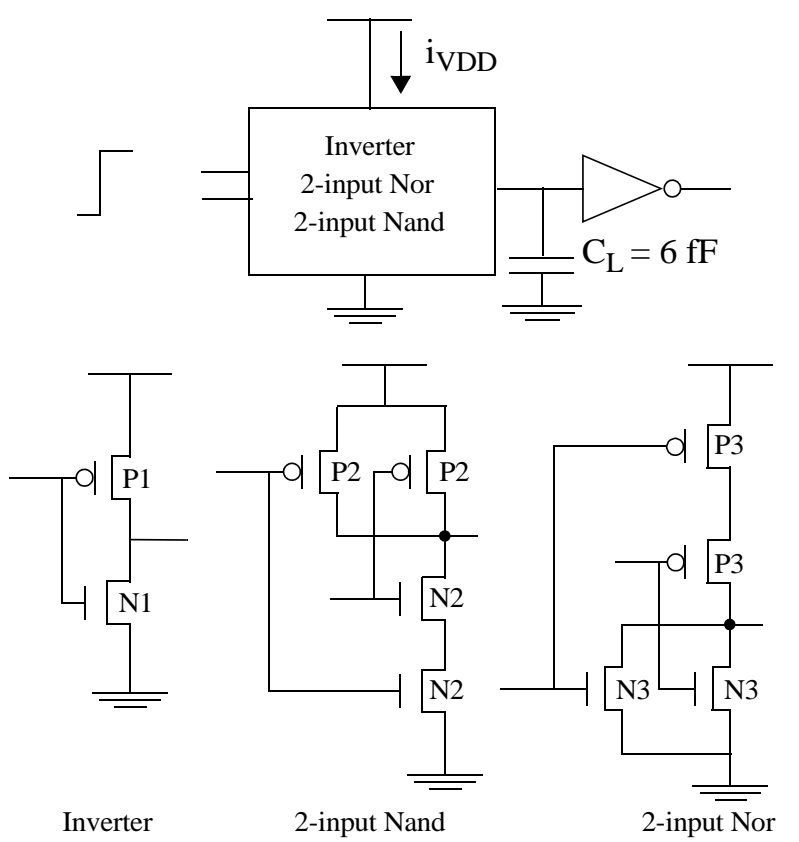

Figure 2. Simulation set-up. Schematic of Inverter, Nand and Nor cell

scaled when the transistors are serially connected: $\mathrm{Wn} /$ $\mathrm{Ln}=0.3 / 0.12$ um (N2 in Fig. 2) and Wp/Lp=0.6/0.12 um (P3 in Fig. 2). The results for the basic gates considering only the variation in $\mathrm{V}_{\mathrm{DD}}$ and excluding the optimization procedure $(f w=1)$ are shown in table I. Data for

TABLE I. SimUlation ResUltS FOR the CiRCUITS OF Fig. 2 Without ANY OptIMIZATION PROCEDURE

\begin{tabular}{|c|c|c|c|c|c|c|}
\hline & $f v d d$ & $f w$ & $\begin{array}{c}\text { tp } \\
(\mathrm{ps})\end{array}$ & $\begin{array}{c}\mathrm{I}_{\mathrm{peak}} \\
(\mathrm{uA})\end{array}$ & $\begin{array}{c}\mathrm{I}_{\text {avg }} \\
(\mathrm{uA})\end{array}$ & $\begin{array}{c}\mathrm{I}_{\text {leakage }} \\
(\mathrm{nA})\end{array}$ \\
\hline \hline \multirow{2}{*}{$\mathrm{I}$} & 1 & 1 & 57.32 & 90.29 & 0.901 & 6.26 \\
\cline { 2 - 7 } $\mathrm{N}$ & $5 / 6$ & 1 & 66.11 & 65.58 & 0750 & 4.83 \\
\cline { 2 - 7 } $\mathrm{V}$ & $2 / 3$ & 1 & 82.75 & 41.75 & 0.601 & 3.69 \\
\cline { 2 - 7 } & $1 / 2$ & 1 & 124.6 & 20.86 & 0.450 & 2.78 \\
\hline \multirow{2}{*}{$\mathrm{N}$} & 1 & 1 & 48.80 & 91.24 & 0.556 & 7.76 \\
\cline { 2 - 7 } $\mathrm{O}$ & $5 / 6$ & 1 & 57.21 & 64.49 & 0.463 & 5.97 \\
\cline { 2 - 7 } & $2 / 3$ & 1 & 73.67 & 41.19 & 0.372 & 4.55 \\
\cline { 2 - 7 } & $1 / 2$ & 1 & 116.7 & 26.22 & 0.277 & 3.43 \\
\hline \multirow{2}{*}{$\mathrm{N}$} & 1 & 1 & 59.11 & 89.85 & 0.537 & 6.59 \\
\cline { 2 - 7 } $\mathrm{A}$ & $5 / 6$ & 1 & 69.75 & 64.23 & 0.444 & 5.19 \\
\cline { 2 - 7 } $\mathrm{N}$ & $2 / 3$ & 1 & 90.12 & 39.94 & 0.355 & 4.06 \\
\cline { 2 - 7 } $\mathrm{D}$ & $1 / 2$ & 1 & 142.5 & 20.01 & 0.265 & 3.15 \\
\hline
\end{tabular}

leakage have been obtained by averaging the values corresponding to different patterns. The leakage values are two orders of magnitude below the average values, because the technology selected to carry out the examples uses high threshold transistors, of reduced leakage. For more advanced technologies with low-threshold transistors, the leakage values are higher, but showing similar trends than those in the table. From the results obtained, it is clear that, as $\mathrm{V}_{\mathrm{DD}}$ decreases, tp increases roughly as eq. (6); $\mathrm{I}_{\text {peak }}$ decreases almost linearly, but quantitatively different as expected from eq. (4), because the multifactorial dependence of $I_{\text {peak }}$ makes that the maximum value happens when $v_{G S} \neq V_{D D} / 2$; $I_{\text {avg }}$ decreases linearly (average power quadratically) as expected; and $\mathrm{I}_{\text {leakage }}$ decreases exponentially. The results are very similar for the three gates considered. Obviously, these results are only due to variations in $\mathrm{V}_{\mathrm{DD}}$, because geometries remain unchanged $\left(f_{w}=1\right)$.

The results obtained after the application of the optimization procedures are shown in table II. The values obtained for $f w$ are shown in the third column, and indicate the overhead in area because this factor is multiplied by the width of all the transistors in the cell. It is clear from the results of the table that the value of $f w$ grows exponentially with $\mathrm{V}_{\mathrm{DD}}$, in order to keep a value of propagation delay almost constant. The behavior for supply current parameters is now quite different, because the simultaneous dependence on $\mathrm{W}$ (now is

TABle II.Simulation Results After the Optimization Process.

\begin{tabular}{|c|c|c|c|c|c|c|}
\hline & $f v d d$ & $f w$ & $\begin{array}{c}\mathrm{tp} \\
(\mathrm{ps})\end{array}$ & $\begin{array}{c}\mathrm{I}_{\text {peak }} \\
(\mathrm{uA})\end{array}$ & $\begin{array}{c}\mathrm{I}_{\text {avg }} \\
(\mathrm{uA})\end{array}$ & $\begin{array}{c}\mathrm{I}_{\text {leakage }} \\
(\mathrm{nA})\end{array}$ \\
\hline \hline \multirow{2}{*}{$\mathrm{I}$} & 1 & 1 & 57.32 & 90.29 & 0.901 & 6.26 \\
\cline { 2 - 7 } $\mathrm{N}$ & $5 / 6$ & 1.19 & 57.37 & 76.97 & 0.762 & 4.96 \\
\cline { 2 - 7 } $\mathrm{V}$ & $2 / 3$ & 1.59 & 57.31 & 67.53 & 0.631 & 4.18 \\
\cline { 2 - 7 } & $1 / 2$ & 2.82 & 57.33 & 61.73 & 0.525 & 4.45 \\
\hline \multirow{2}{*}{$\mathrm{N}$} & 1 & 1 & 48.80 & 91.24 & 0.556 & 7.76 \\
\cline { 2 - 7 } $\mathrm{O}$ & $5 / 6$ & 1.25 & 48.68 & 80.89 & 0.492 & 6.44 \\
\cline { 2 - 7 } & $2 / 3$ & 1.78 & 48.81 & 71.26 & 0.447 & 6.03 \\
\cline { 2 - 7 } & $1 / 2$ & 3.67 & 48.83 & 92.78 & 0.467 & 8.44 \\
\hline \multirow{2}{*}{$\mathrm{N}$} & 1 & 1 & 59.11 & 89.85 & 0.537 & 6.59 \\
\cline { 2 - 7 } $\mathrm{A}$ & $5 / 6$ & 1.25 & 59.26 & 82.23 & 0.470 & 5.92 \\
\cline { 2 - 7 } $\mathrm{N}$ & $2 / 3$ & 1.85 & 59.11 & 76.58 & 0.420 & 6.07 \\
\cline { 2 - 7 } $\mathrm{D}$ & $1 / 2$ & 4.3 & 59.08 & 104 & 0.453 & 9.49 \\
\hline
\end{tabular}


$\mathrm{W} f(w)$ and $\mathrm{V}_{\mathrm{DD}}$. This dependence includes an increment in both leakage, peak and average current, respecting to the equivalent values in table I, because of the increment in W/L, but such increment is very reduced if compared with the gain in operation speed, since it remains constant. This is the main consequence of the optimization process. The trend for average current is almost linear, excepting the operation at low $\mathrm{V}_{\mathrm{DD}}$ $(0.6 \mathrm{~V})$, where the value of average current is higher for the Nand and Nor gates, showing this magnitude a local minimum near $0.8 \mathrm{~V}$. The same happens for the peak current, since a maximum value is located at lowest $\mathrm{V}_{\mathrm{DD}}$ for the Nand and Nor gates. The increment in leakage is also considerable for low $\mathrm{V}_{\mathrm{DD}}$, showing a maximum value for the Nand and Nor gates. For this reason, there is an optimum region of operation conditions near $0.8 \mathrm{~V}$ for those considered magnitudes.

A graphical description of these results are depicted in Fig. 3 for the inverter, Fig. 4 for the 2-input Nand and Fig.5 for the 2-input Nor.

\section{Conclusions}

This paper has presented a simulation-based optimization procedure, allowing the selection of geometries for basic cells, providing reduced values of peak current and both dynamic and leakage power consumption. The procedure has been applied to three basic cells in a 130 $\mathrm{nm}$ technology, increasing the size of their transistors, keeping constant the propagation delay, and saving power (dynamic and leakage), with an additional relative reducing the peaks in supply current.

\section{References}

1. C. Piguet (Ed), Low-Power CMOS Circuits: Technology, Logic Design and CAD Tools, CRC Press, 2006.

2. E. Macii (Ed), Ultra Low-Power Electronics and Design, Kluwer Academic Publishers, 2004.

3. D. Soudris, C. Piguet, C. Goutis (Eds), Designing CMOS Circuits for Low Power, Prentice Hall, 2002

4. X. Aragonès, J. L. González and A. Rubio, Analysis and Solutions for Switching Noise Coupling in mixed-signal ICs. Kluwer Academic Publishers, 1999.

5. S. Donnay and G. Gielen (Eds), Substrate Noise Coupling in Mixed-Signal ASICs, Kluwer Academic Publishers, 2003.

6. D. Ciuplys and P. Larsson-Edefors, "On Maximum Current Estimation in CMOS Digital Circuits", IEEE Int. Conf. on VLSI Design, 2004.

7. A. A. Hamoui and N. Rumin, "An Analytical Model for Current, Delay and Power Analysis of Submicron CMOS Logic Circuits", IEEE Transactions on CAS-II, pp. 999-1007, October 2000.

8. T. Sakurai and A. R. Newton, "Alpha-power Law MOSFET Model and its Applications to CMOS Inverter Delay and other Formulas," IEEE J. of Solid-State Circs., pp. 584-594, April 1990.

9. K. Chen and C. Hu, "Performance and $V_{d d}$ Scaling in deep submicrometer CMOS," IEEE J. of Solid-State Circs., pp. 1586-1589, October 1998.
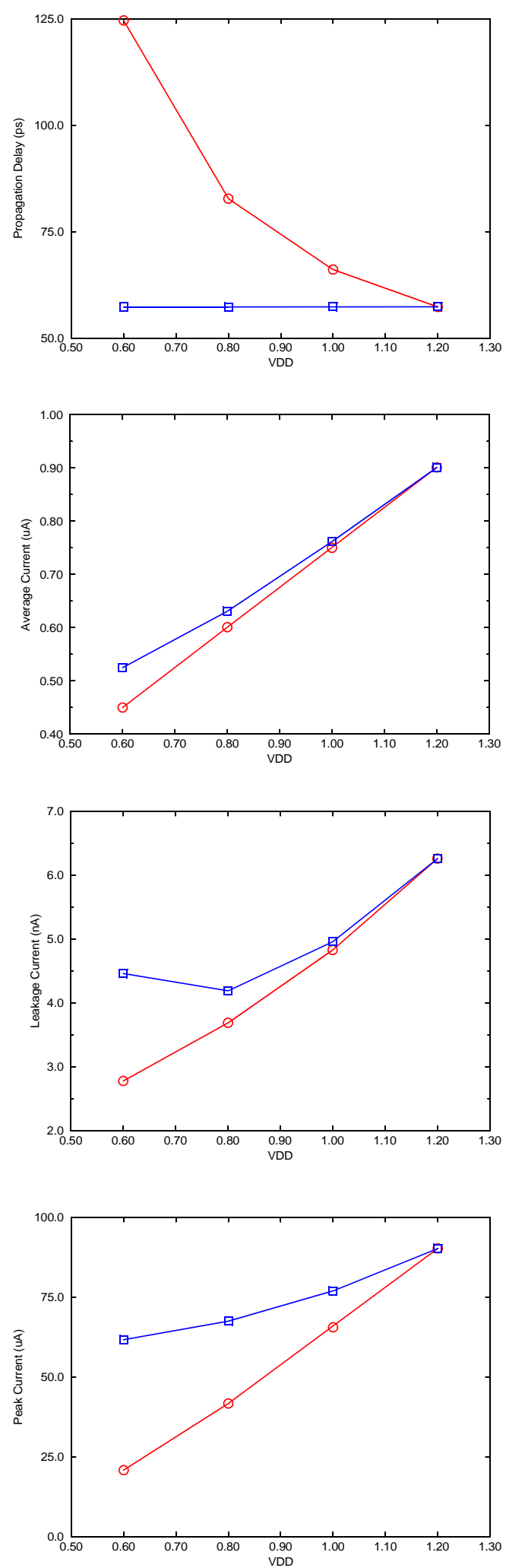

Figure 3.Simulation results for the Inverter, before (circle) and after (square) the optimization process. 

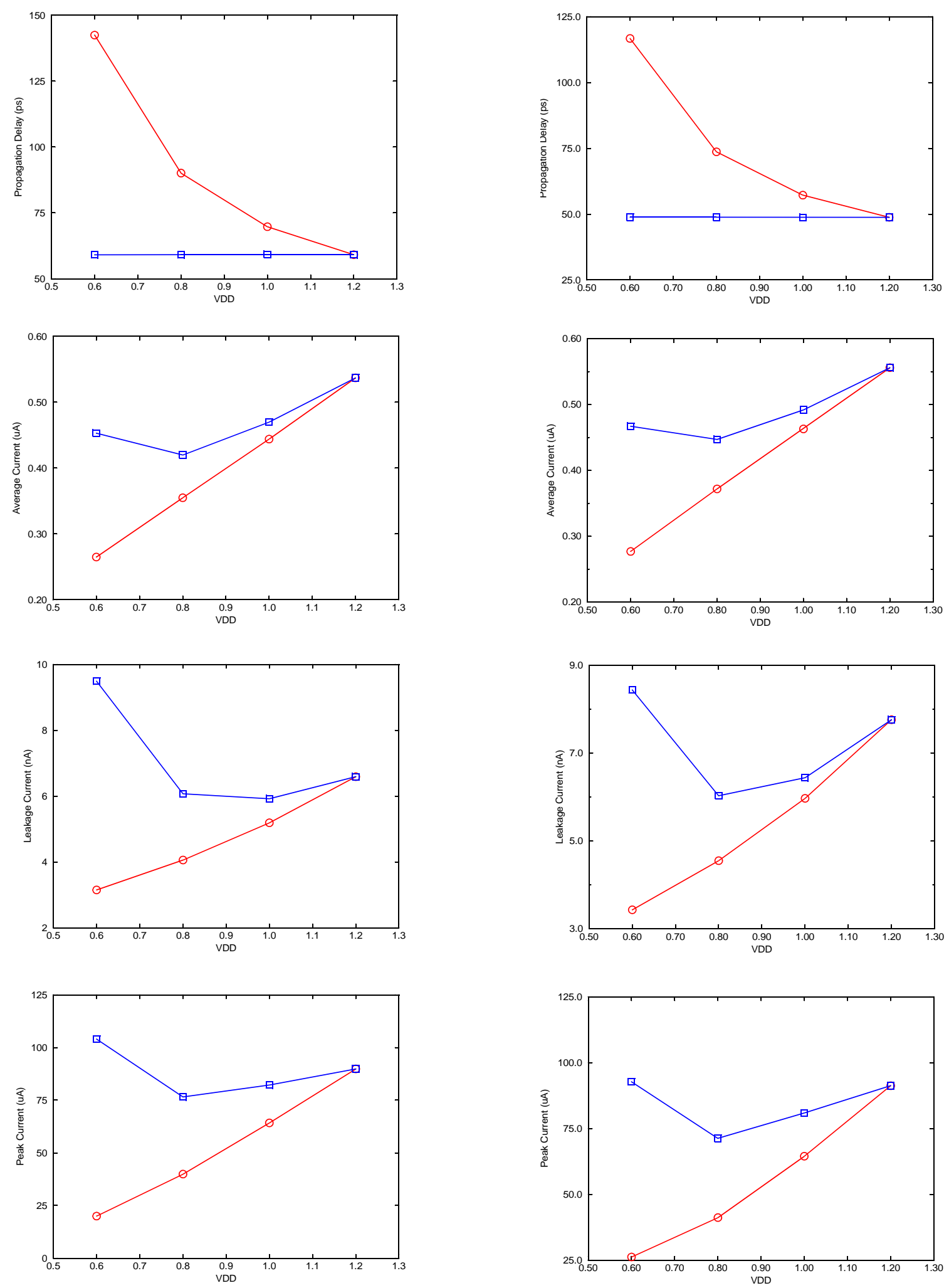

Figure 4.Simulation results for the Nand, before (circle) and after (square) the optimization process.

Figure 5.Simulation results for the Nor, before (circle) and after (square) the optimization process. 